\title{
Fractal analysis - a surrogate technique for material characterization
}

\author{
M. S. Swapna, S. Sankararaman \\ Department of Optoelectronics and Department of Nanoscience and Nanotechnology, University of Kerala, \\ Trivandrum, Kerala, 695581, India. \\ swapnams.opto@gmail.com,drssraman@gmail.com
}

PACS 81.05 U, 05.45.Df, 61.48.De, 81.20.Ka

DOI 10.17586/2220-8054-2017-8-6-809-815

\begin{abstract}
Fractal analysis has emerged as a potential analytical tool in almost all branches of science and technology. The paper is the first report of using fractal dimension as a surrogate technique for estimating particle size. A regression equation is set connecting the soot particle size and fractal dimension, after investigating the Field Emission Scanning Electron Microscopic (FESEM) images of carbonaceous soot from five different sources. Since the fractal dimension is an invariant property under the scale transformation, an ordinary photograph of the soot should also yield the same fractal dimension. This enables one to determine the average size of the soot particles, using the regression equation, by calculating the fractal dimension from the photograph. Hence, instead of frequent measurement of average particle size from FESEM, this technique of estimating the particle size from the fractal dimension of the soot photograph, is found to be a potentially cost-effective and non-contact method.
\end{abstract}

Keywords: fractals, FESEM, carbon nanoparticles, particle size, box-counting.

Received: 16 October 2017

Revised: 26 October 2017

\section{Introduction}

The science of fractal geometry has emerged as a potential analytical tool in almost all branches of science and technology. Fractal analysis is the mathematical method put forward by the polymath Benoit Mandelbrot for quantifying the complex structures and figures [1]. The beauty of this analytical method lies in the fact that it assists in finding the hidden connections and relations existing between different areas of science, mathematics, and art. Fractals are found everywhere in nature $[2,3]$. The scale in which we zoom the image determines the quality of results. The self-replication of objects while zooming differentiates a fractal and a non-fractal object. This technique is used in the chaos theory, to solve the unpredicted problems in an entirely different way, as well as in many dynamic systems [3]. Even though fractals seem complex in nature, they are self-similar structures formed by repetition of simple processes again and again [4,5]. This nature of fractals is greatly exploited in the determination of the benign and malignant breast cancer with higher levels of accuracy and in studying the morphology of galaxies [6-8].

A fractal dimension is the non-integral numerical value which gives the statistical complexity of a fractal image. It is usually expressed as Hausdorff-Besicovitch (HB) dimension $(D)$ which is a mathematical dimension that measures how much space is occupied by the object between Euclidean dimensions [9]. Various methods have been developed for calculating the fractal dimension. Some of the methods are the box-counting method, the successive squares method, the pair-correlation method etc. [10]. In all the methods, the variation of fractal dimensions with the measurement of fractals at different degrees is of concern [1].

Fractal geometry is recently used in the determination of morphological, optical, and thermoelectric characteristics [11] of various nanostructured materials. Such nanostructured materials had been of great interest recently due to their remarkable applications in every field of science and technology. The physical, chemical, electrical, mechanical etc., properties of nanostructured materials are dramatically different when compared with the bulk materials of the same compositions [12-14]. The clusters, molecules, crystallites are various forms of these materials. Carbon nanoparticles are one class among the widely explored nanostructures that have gained significant attention in the twenty-first century as a promising material due to its high thermal and electrical stability, biocompatibility, high tensile strength, etc. The quality and quantity of the CNPs synthesized depends on the raw materials or resources used for the synthesis along with the synthesis procedure adopted. So far, many methods have been developed for the synthesis of CNPs, including laser ablation, chemical and physical vapor deposition, pyrolysis, combustion, electrolysis, ball milling, etc. [15]. The present work describes the synthesis of CNPs by the cost-effective combustion method and a new technique of particle size estimation from the fractal dimension 
of the photograph of the carbon soot. An attempt has also been made to correlate nature of combustion, particle size, structural forms of CNPs, and fractal dimension.

\section{Experimental methods}

To understand whether there is any structural and morphological change in the CNPs in the soot, fractal analysis is conducted. CNPs are synthesized from various sources such as ghee, coconut oil, sesame oil, diesel, and camphor. All these sources are various combinations of saturated, monosaturated and polyunsaturated fatty acids. The high temperatures present during synthesis enables controlled incomplete combustion, resulting in the formation of carbonaceous soot particles.

The samples are purified by liquid phase oxidation method. The sample is mixed with an acidic solution containing nitric acid and sulfuric acid (99.9\%, Sigma-Aldrich) in the ratio 3:1 and ultrasonicated using Scientech SE-366 for 20 minutes. Then the mixture is filtered through a Whatman 41 filter paper, washed four times with distilled water, quenched with ice-cooled water followed by addition of sodium hydroxide for basic neutralization [16]. The resulting mixture is then filtered again using a Whatman 42 filter paper after washing four times with distilled water. These samples are dried and ground in a ball milling unit. The particles are characterized by Nova Nano FESEM. The geometrically and chemically complex structure of soot particles can be analyzed by calculating the fractal dimensions from the scanning electron microscopic images of these nanoparticles.

\subsection{Box counting method}

The most widely used method for obtaining the dimension of self-similar mass fractals [17] in the real world is the box-counting method. In this method, grids of varying sizes $(r)$ are superimposed on the image to be analyzed and the number of boxes containing portions of the image are counted $(N(r)) . N(r)$ is directly proportional to $r^{-D}$. The box-counting dimension is mathematically represented by the logarithmic equation $[1,9]$ :

$$
N(r) \propto r^{-D},
$$

where $D$ is the fractal dimension.

Taking logarithm we get:

$$
\ln N(r)=D \ln \left(\frac{1}{r}\right)+\text { constant }
$$

such that $\lim _{r \rightarrow 0} \frac{\ln N(r)}{\ln (1 / r)}=D$.

From the slope of the $\ln (1 / r)$ vs $\ln N(r)$ graph, the fractal dimension $(D)$ can be calculated.

\section{Results and discussions}

The morphological analysis of the CNPs synthesized from various sources, carried out by the FESEM analysis, reveals the formation of agglomerated nanosized carbon clusters. The FESEM images of the CNPs formed from the combustion of ghee, coconut oil, sesame oil, diesel soot, and camphor are shown in Figs. 1 (a)-(e). The carbon particles obtained ranged from $20-150 \mathrm{~nm}$. The nanospheres in the clusters are bound together by the weak Van der Waal force of attraction. The interaction between the constituent particles was enhanced by any small spatial fluctuations occurring in the samples. This interactive property of soot particles contributes to the formation of aggregates and also results in the restructuring of the CNPs which enables the nanoparticles to show various optical properties. This type of behavior is exhibited by fractals [18]. Depending on the basic source used for the synthesis the size, agglomerating tendency, etc. will also vary. The variation in the fractal dimension with respect to these factors can be studied from the FESEM images.

The SEM images shown in Fig. 1 were subjected to fractal analysis by box counting method and the plots of $\ln N(r)$ vs $\ln (1 / r)$ are shown in Fig. 2. All graphs show high $R^{2}$ value, indicating a high degree of correlation between $N(r)$ and $r$. The fractal dimensions, $R^{2}$ values, and the particle sizes for CNPs obtained from different sources are shown in Table 1.

Varying the fractal dimensions of the CNP agglomerates formed in the soot of various hydrocarbon sources and the size of the agglomerate are shown in Fig. 3. From Fig. 4 it can be seen that the fractal dimension decreased with increasing agglomerate particle size. The fractal dimension depended on the complexity of the system; the greater the complexity, the greater the fractal dimension was. The particle size and fractal dimension $(D)$ showed a strong correlation with $R^{2}$ value equal to one. Hence, a regression equation connecting the two variables - average particle size and fractal dimension - can be set up by fitting a curve to the data by the least squares method. The equation thus set can be used for calculating the particle size from fractal dimension. The closer the value of the 

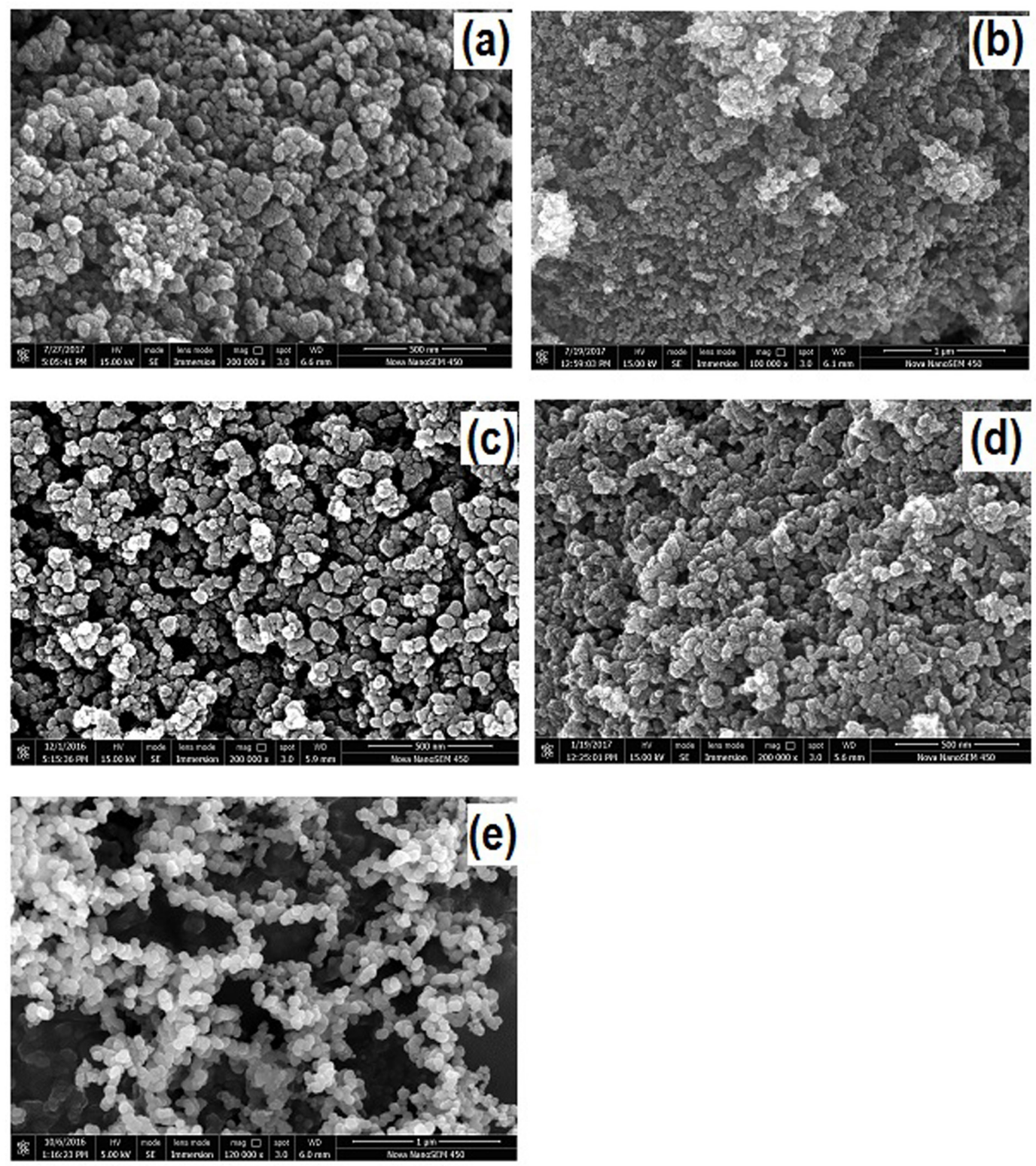

FIG. 1. FESEM images of CNPs obtained from combustion of (a) ghee; (b) coconut oil; (c) sesame oil; (d) diesel; (e) camphor

TABLE 1. Fractal dimensions, $R^{2}$ values, and the particle sizes of the CNPs

\begin{tabular}{|c|c|c|c|c|}
\hline Samples & Fractal dimension $(D)$ & $R^{2}$ value & Particle size $(\mathrm{nm})$ & Average particle size $(\mathrm{nm})$ \\
\hline \hline Ghee & 1.5205 & 0.9703 & $40-90$ & 80 \\
\hline Coconut oil & 1.5541 & 0.9987 & $40-90$ & 65 \\
\hline Sesame oil & 1.6426 & 0.9949 & $25-60$ & 58 \\
\hline Diesel & 1.7805 & 0.9994 & $25-110$ & 43 \\
\hline Camphor & 1.8236 & 0.9932 & $20-60$ & 40 \\
\hline
\end{tabular}



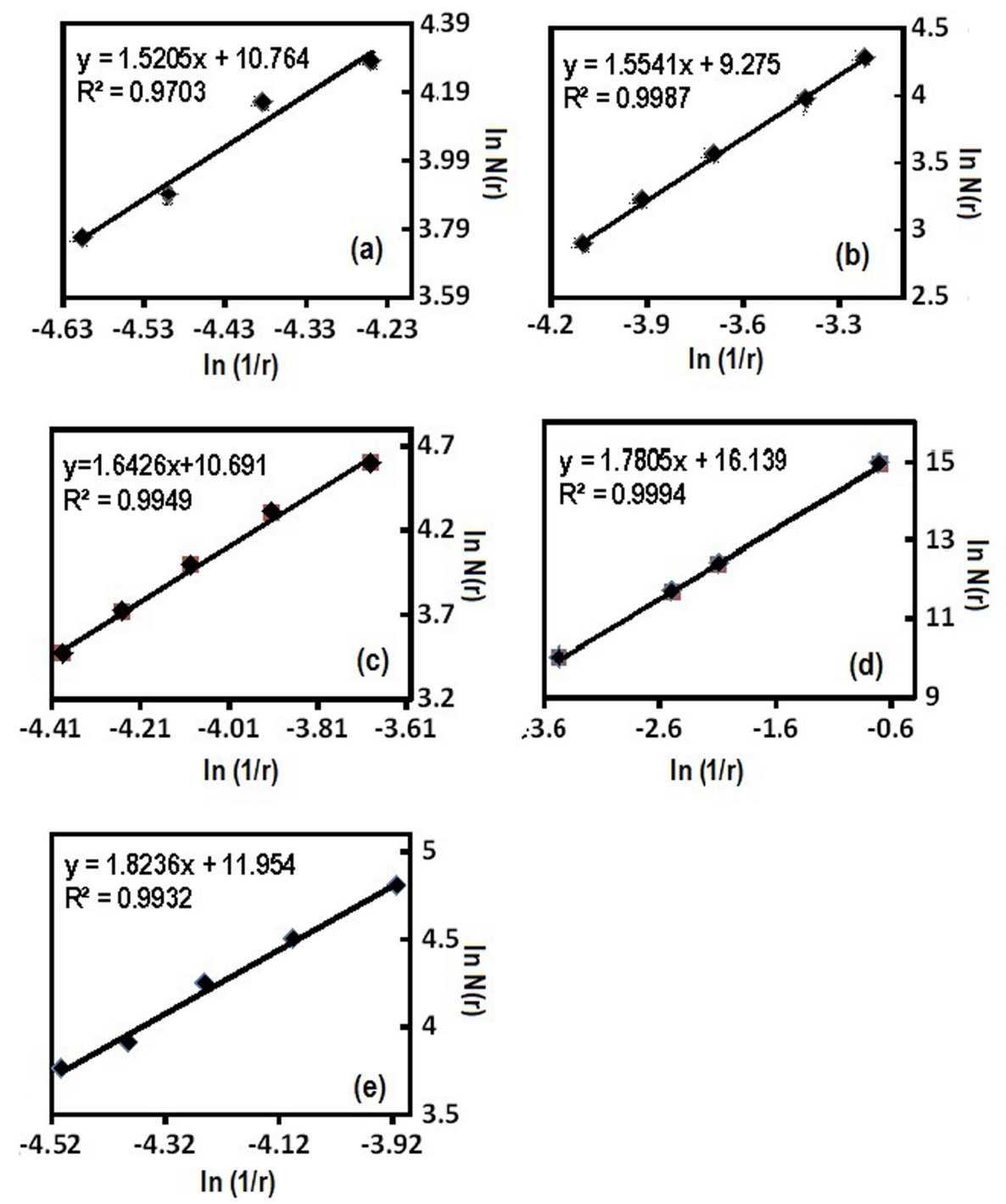

FIG. 2. The plot of $\ln N(r)$ vs $\ln (1 / r)$ for the samples (a) ghee; (b) coconut oil; (c) sesame oil; (d) diesel; (e) camphor

correlation coefficient to one, the greater the accuracy is for estimating the particle size. The equation for the best fit is found to be nonlinear and is given by equation (3):

$$
\text { Particle size }=43298 D^{4}-293413 D^{3}+744612 D^{2}-838785 D+353969 .
$$

From Fig. 4 and Table 1, it can be seen that the average particle size formed during combustion is high in ghee and low in camphor, whereas the fractal dimension is low in ghee and high in camphor. If we analyze the burning process and soot formation, it can be seen that the probability of incomplete combustion is high in ghee, as it does not burn easily as compared to oils. This is why the soot particles from ghee carry larger agglomerates. In the case of camphor, almost complete combustion occurs. Nearly complete combustion leads to the formation of carbon nanotubes, carbon dots and graphene sheets as reported in the literature $[19,20]$. Hence, it can be understood that when the fractal dimension is low, the particle size in the soot is high and there is a lower probability of forming carbon nanotubes and graphene.

In order to understand the possibility of estimating the particle size contained in the soot without recording the FESEM image, the soot particle of all the samples are sprinkled over a white surface, photographed, and fractal dimension is calculated as it should be invariant under scale transformation. The photographic images of the soot samples and their $\ln N(r)$ vs $\ln (1 / r)$ plot are shown in Fig. 5. The fractal dimension obtained from the photographs of soot samples and the particle sizes estimated using equation (3) are given in Table 2. The values thus obtained were compared with the particle sizes estimated from FESEM analysis. 


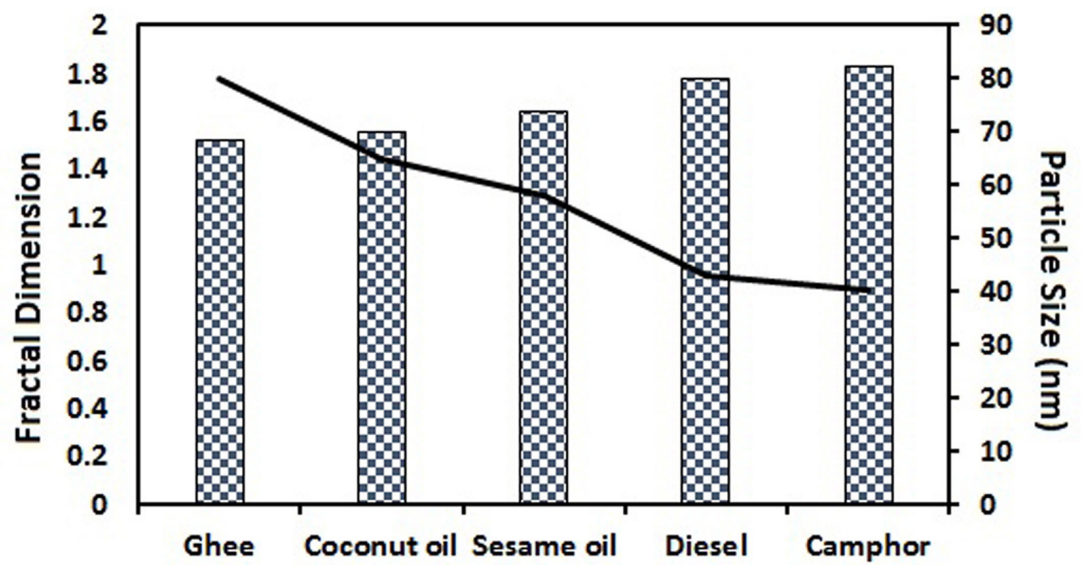

FIG. 3. Fractal dimension and average particle size for various samples

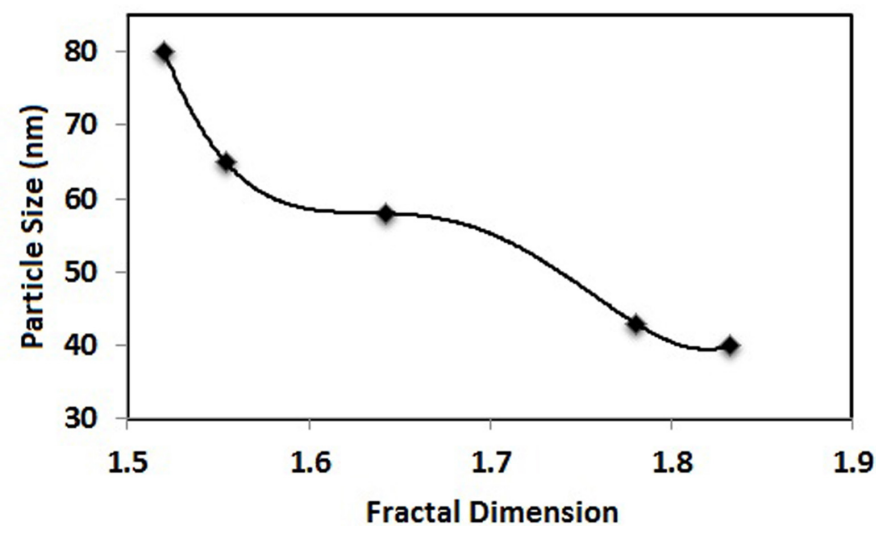

FIG. 4. Variation of particle size with Fractal dimension

TABLE 2. Particle size calculated from fractal dimension of the photograph

\begin{tabular}{|c|c|c|c|}
\hline Sample & Fractal dimension & $\begin{array}{c}\text { Estimated particle size } \\
\text { using Eq. (3) }(\mathrm{nm})\end{array}$ & $\begin{array}{c}\text { Measured average size } \\
\text { from FESEM (nm) }\end{array}$ \\
\hline \hline Ghee & 1.7804 & 41 & $40-90$ \\
\hline Coconut oil & 1.6508 & 57 & $40-90$ \\
\hline Sesame oil & 1.7674 & 44 & $25-60$ \\
\hline Diesel & 1.7763 & 43 & $25-110$ \\
\hline Camphor & 1.7982 & 40 & $20-60$ \\
\hline
\end{tabular}

From Table 2, it can be seen that the particle size calculated from the fractal dimension using equation (3) falls within the range measured from FESEM analysis. This reveals the potential application of estimating the size of CNPs from a photograph without recording the FESEM image, once the regression equation is set. In addition, the fractal dimension gives information regarding the presence of carbon nanotubes, dots, and graphene in the soot. Thus the fractal analysis of the soot can be used for the structural and morphological characterization of soot samples. Though the exact structure cannot be identified, information on the presence of carbon nanotubes, dots, and graphene can be obtained.

\section{Conclusion}

The present work is the first attempt to estimate the particle size from fractals and thus reveals the potential of fractal analysis as a surrogate technique for FESEM analysis. The CNPs present in the soot of five different 
(a)

(b)
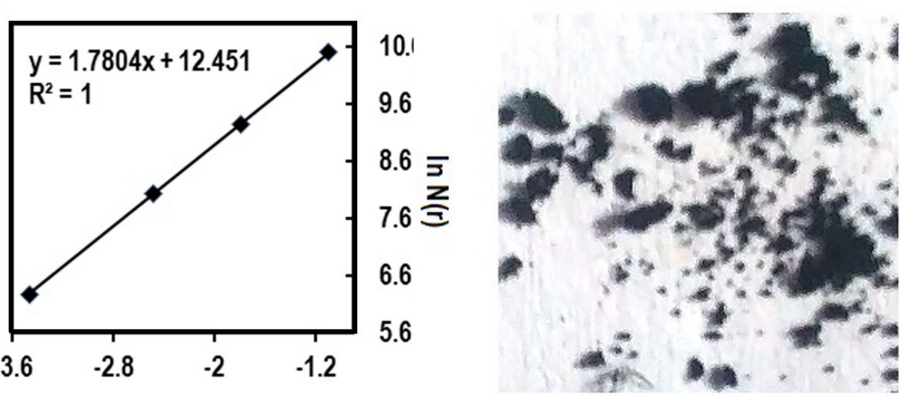

$\ln (1 / r)$

(c)
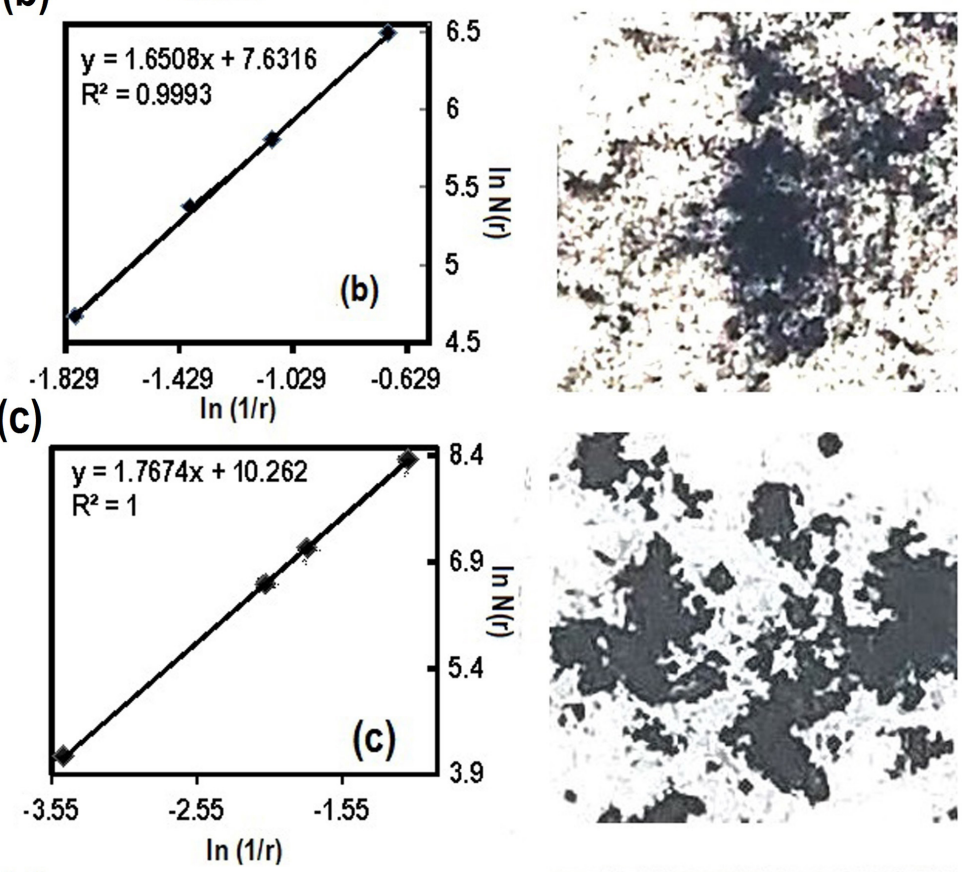

(d)
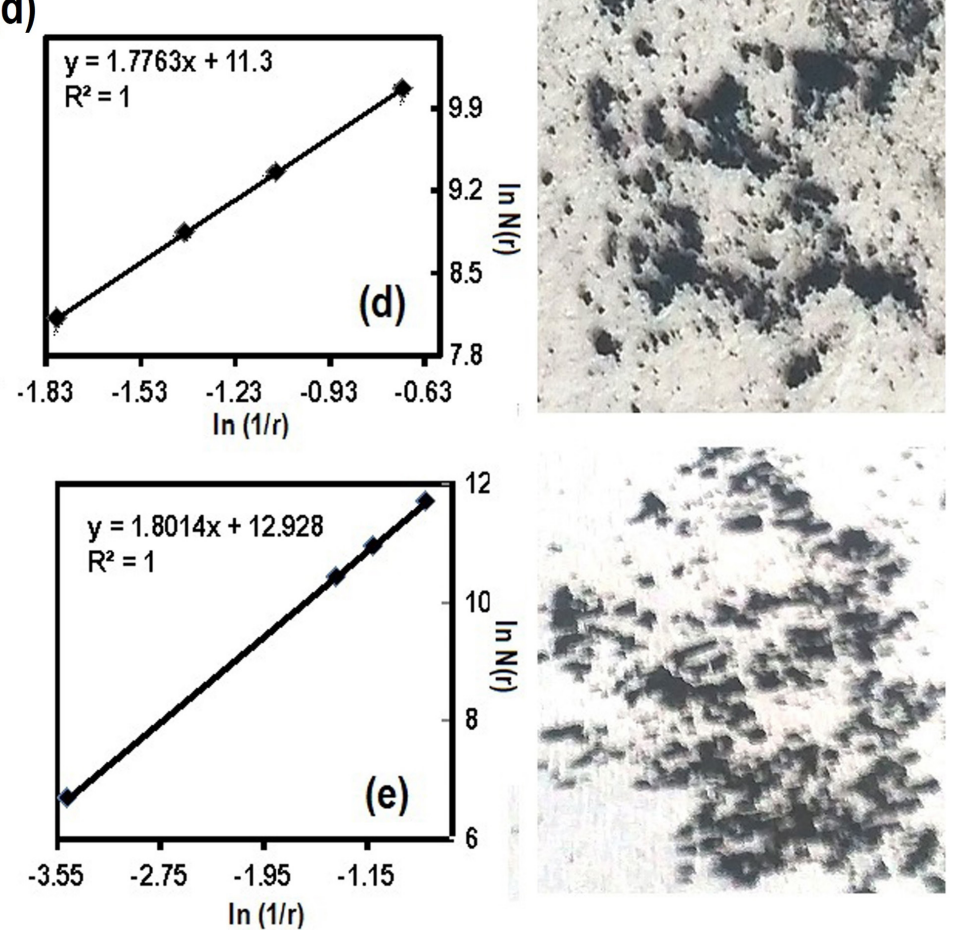

FIG. 5. The photographic image and $\ln N(r)$ vs $\ln (1 / r)$ plot of the soot (a) ghee; (b) coconut oil; (c) sesame oil; (d) diesel; (e) camphor 
sources such as ghee, coconut oil, sesame oil, diesel, and camphor are analyzed to set up the relation connecting particle sizes and fractal dimension. A fourth order polynomial regression equation is set, relating the particle size and fractal dimension with the FESEM images of the soot particles of known particle size. To assess the possibility of estimating the particle size using the regression equation, photographs of soot particles sprinkled over the white surface are recorded and the fractal dimensions are calculated. Results showed that it is possible to estimate the size of the CNP using the regression equation. Thus, the technique is found to be a potentially cost-effective and non-contact method for the estimation of the size of the nanoparticles from ordinary photographs without recording the FESEM image. The fractal dimension is found to be high for soot that has undergone nearly complete combustion, where carbon nanotubes, dots, and graphene are formed. In the case of incomplete combustion, as in ghee, the particle size is high and the fractal dimension is low. Thus, the present method also opens the possibility of exploring the presence of carbon nanotubes, dots and graphene in the sample. However, the method has the limitation of finding the size of isolated particles when there is agglomeration. In such cases, we will obtain the size of the agglomerate. The fractal analysis technique may also be extended to nanoparticles of other materials.

\section{References}

[1] Falconer K. Fractals: A Very Short Introduction. Oxford University press, United Kingdom, 2013.

[2] Kaandorp J.A. Fractal modeling: growth and form in biology. Springer-Verlag, Berlin, Germany, 1994.

[3] Gleick J. Chaos: making a new science. Penguin Books, New York, 1987.

[4] Liebovitch L.S. Fractals and Chaos and Simplified for the Life Sciences, Oxford University Press, 1998.

[5] Liebovitch L.S., Shehadeh L. The Mathematics and Science of Fractals. URL: www. ccs. fau.edu/liebovitch/larry.html.

[6] Dobrescu R., Ichim L., Mocanu S., Popescu D. Benign and Malignant Breast Tumors: Diagnosis Using Fractal Measures. Proceedings of the 18th International Conference on System Theory, IEEE, Control and Computing, Sinaia, Romania, October 17-19, 2014, P. 82-86.

[7] Harrar K., Hamami L. The box counting method for evaluate the fractal Dimension in radiographic images. 6th WSEAS International Conference on Circuits, Systems, Electronics, Control \& Signal Processing, Cairo, Egypt, December 29-31, 2007, P. 385-389.

[8] Seshadri T.R. Fractal analysis of galaxy surveys. Bull. Astr. Soc. India, 2005, 33, P. 1-9.

[9] Addison P.S. Fractals and Chaos: An Illustrated Course. Institute of Physics Publishing, Dirac House, UK, 1997.

[10] Annadhason A. Methods of Fractal Dimension Computation. International Journal of Computer Science and Information Technology \& Security, 2012, 2 (1), P. 166-169.

[11] Tripolskii A.I., Serebrii T.G., et al. Fractal analysis of carbon nanotube agglomerates obtained by chemical vapor decomposition of Ethylene over nickel nanoparticles. Theoretical and Experimental Chemistry, 2009, 45 (2), P. 93-97.

[12] Kumar D., Verma V., Dharamvir K. Elastic Moduli of Carbon Nanotubes Using Second Generation Improved Brenner Potential. J. Nano Res., 2011, 15, P. 1-10.

[13] Anu Mohan, Manoj B. Synthesis and Characterization of Carbon Nanospheres from Hydrocarbon Soot. Int. J. Electrochem. Sci., 2012, 7, P. 9537-9549.

[14] Ungár T., Gubicza J., Gabor R., Cristian Pantea N. Microstructure of carbon blacks determined by X-ray diffraction profile analysis. Carbon, 2002, 4, P. 929-937.

[15] Flahaut E., Peigney A., Laurent C., Rousset A. Synthesis of single-walled carbon nanotubeCoMgO composite powders and extraction of the nanotubes. J. Mater. Chem., 2000, 10, P. 249.

[16] Hussain S., Jha P., et al. Spectroscopic Investigation of Modified Single Wall Carbon Nanotube. J. Mod. Phys., 2011,2 , P. 538-543.

[17] Feder J. Fractals. Plenum Press, New York, 1988.

[18] Markel V.A., George T.F. Optics of Nanostructured Materials. John Wiley \& Sons, 2001.

[19] Swapna M.S., Sankararaman S. Investigation of Graphene Oxide in Diesel Soot. J. Mater. Sci. Nanotechnol., 2017,5 (1), P. 104.

[20] Swapna M.S., Pooja V.M., et al. Synthesis and characterization of carbon nano kajal. JOJ Material Sci., 2017,1 (4), 555566. 